\title{
LINEAR DIFFERENTIAL-DIFFERENCE OPERATORS AND THEIR ADJOINTS ${ }^{1}$
}

\author{
DAVID K. HUGHES
}

Abstract. The formal adjoint for a first order matrix differential-difference operator is shown to be a true Hilbert space adjoint, and conditions under which such operators are selfadjoint (in a Hilbert space sense) are derived. Differential-difference operators whose domains are defined by a given initial function cannot be selfadjoint, whereas certain operators whose domains are defined only by conditions at the end points of an interval can be selfadjoint.

In the past several years there has been considerable interest in differential-difference equations (hereafter abbreviated by $D$. D. equations) or differential equations with retarded argument. A large majority of the publications in this area have dealt with existence theory or stability theory for such equations. There seem to have been very few results in the direction of boundary value problems and adjoints for a D. D. equation. A. Halanay has given some results concerned with boundary value problems in [4]. Also both he and $\mathrm{R}$. Bellman and K. Cooke use the formal adjoint of a D. D. equation to obtain an analog of the variations of parameter formula [3, pp. $359-362]$ and [2, pp. 320-323].

This article considers the problem of determining an adjoint of a D. D. equation from the point of view of linear operators in Hilbert space. We define two distinct operators, and in particular we investigate the possibility of their being selfadjoint as operators on a dense subset of a Hilbert space. As far as is known to the author, this is the first paper in which it is shown that the formal adjoint is also a Hilbert space adjoint.

Equations like those which we consider arise as Euler-type equations for variational problems with delayed argument having quadratic in tegrand. See for example Hughes [5].

Throughout this paper $A_{i}, B_{i}$ denote continuous $n$ by $n$ matrices defined on $[a-\tau, b+\tau]$ where $\tau$ represents a positive real number.

Presented to the Society, January 23, 1969; received by the editors April 18, 1969.

AMS 1969 subject classifications. Primary 3475; Secondary 3430.

Key words and phrases. Differential-difference operators, differential-difference equations, functional-differential equations, formal adjoint, Hilbert space adjoint, selfadjoint differential operator.

1 This research was supported by the Development Committee for Science and Mathematics of Abilene Christian College. 
We also suppose that $M$ and $N$ are $m$ by $n$ constant matrices such that the matrix $(M: N)$ is of rank $m, m \leqq 2 n$. A prime will always denote differentiation.

Problem I. Let $H_{0}$ be the Hilbert space of $n$-dimensional vector functions $x$ defined on $[a-\tau, b+\tau]$ whose components are in $L^{2}$ and $x(t)=0, a-\tau \leqq t<a$. The inner product is given by $\langle x, y\rangle=\int_{a}^{b+\tau} y^{*} x$ where $y^{*}$ is the transpose of $y$.

Define the boundary operator $\mu(x)$ and the differential-difference expression $E(x)$ by the following formulas:

$$
\begin{aligned}
\mu(x)= & M x(a)+N x(b+\tau), \\
E(x)= & A_{0}(t) \dot{x}(t)+A_{1}(t) \dot{x}(t-\tau)+A_{2}(t) \dot{x}(t+\tau) \\
& +B_{0}(t) x(t)+B_{1}(t) x(t-\tau)+B_{2}(t) x(t+\tau), \quad a \leqq t \leqq b, \\
E(x)= & A_{0}(t) \dot{x}(t)+A_{1}(t) \dot{x}(t-\tau)+B_{0}(t) x(t)+B_{1}(t) x(t-\tau),
\end{aligned}
$$$$
b \leqq t \leqq b+\tau,
$$

where $A_{1}, A_{2}, B_{1}, B_{2}$ are not all zero.

We denote by $D$ the set of all $x$ in $H_{0}$ with the following properties:

(i) $x$ is absolutely continuous on $[a, b+\tau]$.

(ii) $E(x)$ is in $\boldsymbol{H}_{\mathbf{0}}$.

Let us now define a differential-difference operator $L: D \rightarrow H_{0}$ by setting $L x=E(x)$ for all $x$ in $D$.

Since $D$ is dense in $H_{0}$, there is a well-defined adjoint operator $L^{*}$.

THEOREM 1. If $z$ is in the domain of $L^{*}$, then

$$
\begin{array}{rlr}
L^{*} z= & -\left[A_{0}^{*}(t) z(t)+A_{1}^{*}(t+\tau) z(t+\tau)\right]^{\prime}+\left[B_{0}^{*}(t) z(t)+B_{1}^{*}(t+\tau) z(t+\tau)\right], \\
L^{*} z= & -\left[A_{0}^{*}(t) z(t)+A_{1}^{*}(t+\tau) z(t+\tau)+A_{2}^{*}(t-\tau) z(t-\tau)\right]^{\prime} & a \leqq t<a+\tau ; \\
& +\left[B_{0}^{*}(t) z(t)+B_{1}^{*}(t+\tau) z(t+\tau)+B_{2}^{*}(t-\tau) z(t-\tau)\right], & a+\tau \leqq t<b ; \\
L^{*} z=- & {\left[A_{0}^{*}(t) z(t)+A_{2}^{*}(t-\tau) z(t-\tau)\right]^{\prime}+\left[B_{0}^{*}(t) z(t)+B_{2}^{*}(t-\tau) z(t-\tau)\right],} \\
& b \leqq t \leqq b+\tau .
\end{array}
$$

Let $D_{0}$ be the set of all $x$ in $D$ such that

$$
x(a)=0=x(b)=x(b+\tau)=x(a+\tau) .
$$

Let $x$ be in $D_{0}$ and solve the equation

$$
\langle L x, z\rangle=\langle x, \theta\rangle \text { for } \theta .
$$




$$
\begin{aligned}
0= & \int_{a}^{b} z^{*}(t)\left[A_{0}(t) \dot{x}(t)+A_{1}(t) \dot{x}(t-\tau)+A_{2}(t) \dot{x}(t+\tau)\right. \\
& \left.\quad+B_{0}(t) x(t)+B_{1}(t) x(t-\tau)+B_{2}(t) x(t+\tau)\right] d t \\
& -\int_{a}^{b+\tau} \theta^{*} x(t) d t \quad \\
& +\int_{b}^{b+r} z^{*}(t)\left[A_{0}(t) \dot{x}(t)+A_{1}(t) \dot{x}(t-\tau)\right. \\
& \left.\quad+B_{0}(t) x(t)+B_{1}(t) x(t-\tau)\right] d t .
\end{aligned}
$$

In the terms involving $x(t-\tau)$ and $\dot{x}(t-\tau)$ let $s=t-\tau$. Make the substitution $s=t+\tau$ in the terms with $x(t+\tau)$ and $\dot{x}(t+\tau)$. The preceding expression then becomes

$$
\begin{aligned}
& 0=\int_{a}^{a+\tau}\left\{\left[z^{*}(t) A_{0}(t)+z^{*}(t+\tau) A_{1}(t+\tau)\right] \dot{x}(t)\right. \\
& \left.+\left[z^{*}(t) B_{0}(t)+z^{*}(t+\tau) B_{1}(t+\tau)-\theta^{*}\right] x(t)\right\} d t \\
& +\int_{a+\tau}^{b}\left\{\left[z^{*}(t) A_{0}(t)+z^{*}(t+\tau) A_{1}(t+\tau)+z^{*}(t-\tau) A_{2}(t-\tau)\right] \dot{x}(t)\right. \\
& +\left[z^{*}(t) B_{0}(t)+z^{*}(t+\tau) B_{1}(t+\tau)\right. \\
& \left.\left.+z^{*}(t-\tau) B_{2}(t-\tau)-\theta^{*}\right] x(t)\right\} d t \\
& +\int_{b}^{b+\tau}\left\{\left[z^{*}(t) A_{0}(t)+z^{*}(t-\tau) A_{2}(t-\tau)\right] \dot{x}(t)\right. \\
& \left.+\left[z^{*}(t) B_{0}(t)+z^{*}(t-\tau) B_{2}(t-\tau)-\theta^{*}\right] x(t)\right\} d t .
\end{aligned}
$$

Integrating by parts and noting that $x$ is in $D_{0}$, we have the following equation: $0=\int_{a}^{b+\tau} \psi(t) \dot{x}(t) d t$ where

$$
\begin{aligned}
& \psi(t)=z^{*}(t) A_{0}(t)+z^{*}(t+\tau) A_{1}(t+\tau) \\
& -\int_{a}^{t}\left[z^{*}(s) B_{0}(s)+z^{*}(s+\tau) B_{1}(s+\tau)-\theta^{*}\right] d s, \quad a \leqq t<a+\tau, \\
& \psi(t)=z^{*}(t) A_{0}(t)+z^{*}(t+\tau) A_{1}(t+\tau)+z^{*}(t-\tau) A_{2}(t-\tau) \\
& -\int_{a+\tau}^{t}\left[z^{*}(s) B_{0}(s)+z^{*}(s+\tau) B_{1}(s+\tau)\right. \\
& \left.+z^{*}(s-\tau) B_{2}(s-\tau)-\theta^{*}\right] d s, \quad a+\tau \leqq t<b,
\end{aligned}
$$




$$
\begin{aligned}
\psi(t)= & z^{*}(t) A_{0}(t)+z^{*}(t-\tau) A_{2}(t-\tau) \\
& -\int_{b}^{t}\left[z^{*}(s) B_{0}(s)+z^{*}(s-\tau) B_{2}(s-\tau)-\theta^{*}\right] d s, \quad b \leqq t \leqq b+\tau .
\end{aligned}
$$

Now the fundamental lemma of the calculus of variations yields that $\psi$ is constant on each of the subintervals $(a, a+\tau),(a+\tau, b)$, and $(b, b+\tau)$. Upon differentiation of $\psi$ and solution for $\theta$, we arrive at the result of the theorem.

The result of Theorem 1 shows that the operator $L$ defined by $L x=E(x)$ on $D$ cannot be selfadjoint. However, using this result as a guide, we now consider a $D$. D. operator whose adjoint has the same form as the original operator.

Problem II. Let $H$ be the Hilbert space of all $n$-dimensional vector functions defined on $[a-\tau, b+\tau]$ whose components are square integrable. The inner product is given by $\langle x, y\rangle=\int_{a-\tau}^{b+\tau} y * x$.

Define the boundary operator $\lambda$ by the equation

$$
\lambda(x)=M x(a-\tau)+N x(b+\tau)
$$

and define the symbol $D(x)$ by the following $\mathrm{D}$. D. expressions:

$$
\begin{aligned}
D(x)= & A_{0}(t) \dot{x}(t)+A_{2}(t) \dot{x}(t+\tau)+B_{0}(t) x(t)+B_{2}(t) x(t+\tau), & & a-\tau \leqq t<a ; \\
D(x)= & A_{0}(t) \dot{x}(t)+A_{1}(t) \dot{x}(t-\tau)+A_{2}(t) \dot{x}(t+\tau) & & \\
& +B_{0}(t) x(t)+B_{1}(t) x(t-\tau)+B_{2}(t) x(t+\tau), & & a \leqq t<b ; \\
D(x)= & A_{0}(t) \dot{x}(t)+A_{1}(t) \dot{x}(t-\tau)+B_{0}(t) x(t)+B_{1}(t) x(t-\tau), & & b \leqq t \leqq b+\tau .
\end{aligned}
$$

Let $B$ be the set of all $x$ in $H$ which satisfy the conditions:

(i) $x$ is absolutely continuous on $[a-\tau, b+\tau]$.

(ii) $D(x)$ is in $H$.

We now define an operator $A$ on $B$ to $H$ by the formula $A x=D(x)$ for $x$ in $B$. Since $B$ is dense in $H$, there exists a well-defined adjoint operator $A^{*}$. Let $B^{*}$ be the domain of $A^{*}$.

THEOREM 2. If $z$ is in $B^{*}$, then

$$
\begin{array}{rlr}
A^{*} z= & -\left[A_{0}^{*}(t) z(t)+A_{1}^{*}(t+\tau) z(t+\tau)\right]^{\prime}+B_{0}^{*}(t) z(t)+B_{1}^{*}(t+\tau) z(t+\tau), \\
A^{*} z= & -\left[A_{0}^{*}(t) z(t)+A_{1}^{*}(t+\tau) z(t+\tau)+A_{2}^{*}(t-\tau) z(t-\tau)\right] & a-\tau \leqq t \leqq a, \\
& +B_{0}^{*}(t) z(t)+B_{1}^{*}(t+\tau) z(t+\tau)+B_{2}^{*}(t-\tau) z(t-\tau), & a \leqq t \leqq b, \\
A^{*} z= & -\left[A_{0}^{*}(t) z(t)+A_{2}^{*}(t-\tau) z(t-\tau)\right]^{\prime}+B_{0}^{*}(t) z(t)+B_{2}^{*}(t-\tau) z(t-\tau), \\
& & b \leqq t \leqq b+\tau .
\end{array}
$$


Again we solve the equation $0=\langle A x, z\rangle-\langle x, \theta\rangle$ for $\theta$. We must again restrict $x$ to the set $B_{0}=\{x$ in $B: x(a-\tau)=x(a)=x(b)=x(b+\tau)=0\}$. The calculations are quite analogous to those used in the proof of Theorem 1.

Let $B^{0}$ be the set of all $x$ in $B$ with the property that $\lambda(x)=0$. Let $B^{0}$ be the domain of $A$ and let $B^{0 *}$ be the domain of $A^{*}$.

THEOREM 3. If $z$ is in $B^{0 *}$, then $z$ satisfies the following boundary conditions:

$$
\begin{aligned}
& A_{0}^{*}(a-\tau) z(a-\tau)+A_{1}^{*}(a) z(a)=-M^{*} \sigma \\
& A_{0}^{*}(b+\tau) z(b+\tau)+A_{2}^{*}(b) z(b)=N^{*} \sigma
\end{aligned}
$$

where $\sigma$ is an m-dimensional vector.

Let $x$ be in $B$ and $z$ in $B^{*}$. Then

$$
\begin{aligned}
& \langle A x, z\rangle-\left\langle x, A^{*} z\right\rangle \\
& =\int_{a \rightarrow \tau}^{a}\left\{z^{*}(t)\left[A_{0}(t) \dot{x}(t)+A_{2}(t) \dot{x}(t+\tau)+B_{0}(t) x(t)+B_{2}(t) x(t+\tau)\right]\right. \\
& +\left\{\left[z^{*}(t) A_{0}(t)+z^{*}(t+\tau) A_{1}(t+\tau)\right]^{\prime}\right. \\
& \left.\left.-z^{*}(t) B_{0}(t)-z^{*}(t+\tau) B_{1}(t+\tau)\right\} x(t)\right\} d t \\
& +\int_{a}^{b}\left\{z ^ { * } ( t ) \left[A_{0}(t) \dot{x}(t)+A_{1}(t) \dot{x}(t-\tau)+A_{2}(t) \dot{x}(t+\tau)\right.\right. \\
& \left.+B_{0}(t) x(t)+B_{1}(t) x(t-\tau)+B_{2}(t) x(t+\tau)\right] \\
& +\left\{\left[z^{*}(t) A_{0}(t)+z^{*}(t+\tau) A_{1}(t+\tau)+z^{*}(t-\tau) A_{2}(t-\tau)\right]^{\prime}\right. \\
& \begin{array}{r}
\left.\left.-z^{*}(t) B_{0}(t)-z^{*}(t+\tau) B_{1}(t+\tau)-z^{*}(t-\tau) B_{2}(t-\tau)\right\} x(t)\right\} d t \\
+\int_{b}^{b+\tau}\left\{z^{*}(t)\left[A_{0}(t) \dot{x}(t)+A_{1}(t) \dot{x}(t-\tau)+B_{0}(t) x(t)+B_{1}(t) x(t-\tau)\right]\right.
\end{array} \\
& +\left\{\left[z^{*}(t) A_{0}(t)+z^{*}(t-\tau) A_{2}(t-\tau)\right]^{\prime}\right. \\
& \left.\left.-z^{*}(t) B_{0}(t)-z^{*}(t+\tau) B_{1}(t+\tau)\right\} x(t)\right\} d t \\
& =\int_{a-\tau}^{a}\left\{\left[z^{*}(t) A_{0}(t)+z^{*}(t+\tau) A_{1}(t+\tau)\right] x(t)\right\}^{\prime} d t \\
& +\int_{a}^{b}\left\{\left[z^{*}(t) A_{0}(t)+z^{*}(t+\tau) A_{1}(t+\tau)\right.\right. \\
& \left.\left.+z^{*}(t-\tau) A_{2}(t-\tau)\right] x(t)\right\}^{\prime} d t \\
& +\int_{b}^{b+\tau}\left\{\left[z^{*}(t) A_{0}(t)+z^{*}(t-\tau) A_{2}(t-\tau)\right] x(t)\right\}^{\prime} d t .
\end{aligned}
$$


Now we have the equations

$$
\begin{aligned}
0= & {\left[z^{*}(a-) A_{0}(a)+z^{*}(a+\tau-) A_{1}(a+\tau)\right] x(a) } \\
& -\left[z^{*}(a-\tau) A_{0}(a-\tau)+z^{*}(a+) A_{1}(a)\right] x(a-\tau) \\
& +\left[z^{*}(b-) A_{0}(b)+z^{*}(b+\tau) A_{1}(b+\tau)+z^{*}(b-\tau-) A_{2}(b-\tau)\right] x(b) \\
& -\left[z^{*}(a+) A_{0}(a)+z^{*}(a+\tau+) A_{1}(a+\tau)+z^{*}(a-\tau) A_{2}(a-\tau)\right] x(a) \\
& +\left[z^{*}(b+\tau) A_{0}(b+\tau)+z^{*}(b-) A_{2}(b)\right] x(b+\tau) \\
& -\left[z^{*}(b+) A_{0}(b)+z^{*}(b-\tau+) A_{2}(b-\tau)\right] x(b) \\
= & {\left[z^{*}(a-) A_{0}(a)+z^{*}(a+\tau-) A_{1}(a+\tau)-z^{*}(a+) A_{0}(a)\right.} \\
& \left.\quad-z^{*}(a+\tau+) A_{1}(a+\tau)-z^{*}(a-\tau) A_{2}(a-\tau)\right] x(a) \\
& +\left[z^{*}(b-) A_{0}(b)+z^{*}(b+\tau) A_{1}(b+\tau)+z^{*}(b-\tau-) A_{2}(b-\tau)\right. \\
& \left.\quad-z^{*}(b+) A_{0}(b)-z^{*}(b-\tau+) A_{2}(b-\tau)\right] x(b) \\
& +\left[z^{*}(b+\tau) A_{0}(b+\tau)+z^{*}(b-) A_{2}(b)\right] x(b+\tau) \\
& -\left[z^{*}(a-\tau) A_{0}(a-\tau)+z^{*}(a+) A_{1}(a)\right] x(a-\tau) .
\end{aligned}
$$

We conclude that each of the first two expressions in brackets is zero as well as

$$
\begin{aligned}
& {\left[z^{*}(b+\tau) A_{0}(b+\tau)+z^{*}(b-) A_{2}(b)\right] x(b+\tau)} \\
& -\left[z^{*}(a-\tau) A_{0}(a-\tau)+z^{*}(a+) A_{1}(a)\right] x(a-\tau)=0 .
\end{aligned}
$$

Hence there exists a constant $m$-dimensional vector $\sigma$ such that

$$
z^{*}(a-\tau) A_{0}(a-\tau)+z^{*}(a+) A_{1}(a)=-\sigma^{*} M
$$

and

$$
z^{*}(b+\tau) A_{0}(b+\tau)+z^{*}(b-) A_{2}(b)=\sigma^{*} N .
$$

The conclusion of the theorem follows by transposition.

It is to be noted that as a result of the proof of Theorem 3, we find that the differentiated portion of the operator $A^{*}$ is absolutely continuous on $[a-\tau, b+\tau]$.

Also as a result of Theorem 3 , we find that $A$ cannot be selfadjoint. However if $A_{1}=A_{2}=0$ on $[a-\tau, b+\tau]$, then $A$ has the possibility of being selfadjoint. The following theorem is proved as in Reid [6].

THEOREM 4. The operator $A$ with $A_{1}=A_{2}=0$ is selfadjoint if and only if $A_{0}=-A_{0}^{*}, B_{0}=B_{0}^{*}-A_{0}^{* \prime}, \quad B_{1}^{*}(t+\tau)=B_{2}(t)$, and $M A_{0}^{*}(a-\tau)^{-1} M^{*}=N A_{0}^{*}(b+\tau)^{-1} N^{*}$. 


\section{BIBLIOGRAPHY}

1. H. T. Banks, Represenlations for solutions of linear functional differential equations, J. Differential Equations 5 (1969), 399-409. MR 38 \#3547.

2. R. Bellman and K. Cooke, Differential-difference equations, Academic Press, New York, 1963. MR 26 \#5259; errata 27 p. 1399.

3. A. Halanay, Differential equations: Stability, oscillations, time lags, Academic Press, New York, 1966. MR 35 \#6938.

4. - On a boundary-value problem for linear systems with time lag, J. Differential Equations 2 (1966), 47-56. MR 32 \#7902.

5. D. K. Hughes, Variational and optimal control problems with delayed argument, J. Optimization Theory Appl. 2 (1968), 1-14. MR 39 \#4725.

6. W. T. Reid, $A$ class of two-point boundary problems, Illinois J. Math. 2 (1958), 434-453. MR 20 \#3331.

Abilene Christian College, Abilene, Texas 79601 